Research Article

\title{
Screening based on incidence of severe retinopathy of prematurity in a tertiary care center in India: are Indian infants different?
}

\author{
Umamaheswari Balakrishnan, Shafi Jan Mohammad Shaik, Nivetha Manian*, \\ Muthayya Muthukumar, Mary Thomas, Prakash Amboiram, Binu Ninan, Ashok \\ Chandrasekaran, Saravanan Ramaswamy
}

Department of Neonatology, Sri Ramachandra Medical College and Research Institute, Porur, Chennai, India

Received: 08 July 2016

Accepted: 18 July 2016

*Correspondence:

Dr. Nivetha Manian,

E-mail: neosrmc@gmail.com

Copyright: (C) the author(s), publisher and licensee Medip Academy. This is an open-access article distributed under the terms of the Creative Commons Attribution Non-Commercial License, which permits unrestricted non-commercial use, distribution, and reproduction in any medium, provided the original work is properly cited.

\section{ABSTRACT}

Background: Incidence of retinopathy of prematurity (ROP) and screening criteria among the preterm infants varies widely. The incidence of ROP and severe ROP in different Birth Weight (BW) and Gestational Age (GA) groups were studied. The aim of the study was to review the existing screening criteria for ROP based on the highest birth weight and or gestational age of infants who developed severe ROP.

Methods: This is a retrospective descriptive study of neonates with GA of $\leq 34$ weeks and/or BW of $\leq 1750 \mathrm{gm}$ screened for ROP by experienced ophthalmologist from January, 2011 to December, 2015. End point was either complete vascularisation or need for laser therapy as per ETROP guidelines. Severe ROP was defined as ROP needing treatment. Highest BW and or GA of infants who developed severe ROP were taken as cut off points for revising the existing screening criteria for ROP.

Results: Among 1366 infants included in this study, 252 (18.4\%) and 86 (6.2\%) developed ROP and severe ROP, respectively. Mean GA was 29.2 \pm 1.4 weeks and $28.7 \pm 2.3$ among infants with ROP and severe ROP, respectively. Stratified analysis showed a significant increase in the incidence of ROP with decreasing BW and GA $(p<0.001)$. A threshold of $\mathrm{BW} \leq 1750 \mathrm{gm}$ and or GA $\leq 34$ weeks would not miss any infant with severe ROP. Applying screening criteria of developed nations would miss 9.3 to $11.6 \%$ of infants with severe ROP.

Conclusions: We suggest screening for ROP in all premature infants born with GA of $\leq 32$ weeks and or BW of $\leq 1750 \mathrm{gm}$, as this screening criteria has detected all infants with severe ROP.

Keywords: Retinopathy of prematurity, Screening criteria, Incidence, India, Severe retinopathy of prematurity

\section{INTRODUCTION}

Retinopathy of prematurity (ROP) is one of the leading causes of preventable blindness in children. ${ }^{1}$ There has been a growing concern about blindness due to ROP in middle and low income countries and this surge has been described as the third epidemic of ROP. ${ }^{2}$ With the advancement of neonatal care, the survival of premature infants has improved in India but parallel development of human resources in terms of trained ophthalmologist and infrastructure in terms of availability of advanced equipment for treatment, remains a challenge. The targets of vision 2020 include screening for ROP in India and Latin America. ${ }^{3}$ Incidence of ROP varies widely depending on the population screened for ROP and ranges from $29 \%$ to $68 \%$ in developed country and 38 $51 \%$ in India. $^{4-8}$ 
Based on population studies, developed nations have established guidelines for screening ROP. ${ }^{9,10}$ These criteria have been constantly revisited and revised as the need for screening population varies from time to time. ${ }^{11,12}$ Screening criteria needs to be safe as well as efficient so that none of the infants needing ROP treatment are missed and too many infants are not screened unnecessarily. Availability of trained ophthalmologist and the nature of procedure being painful and distressing to infants warrant efficient screening criteria. As severe ROP has been reported in bigger and more mature infants in developing countries the same screening criteria cannot be applied to India. ${ }^{13-15}$ National neonatology forum (NNF), India recommends performance of screening in all preterm infants born $<34$ weeks gestation and or $<1750$ grams birth weight and screening infants between 34 and $36+6$ weeks gestation or 1750 and 2000 birth weight with risk factors for ROP. ${ }^{16}$ Screening criteria was largely based on studies from ophthalmological referral institutes which may not represent general population.

The incidence of ROP and ROP needing treatment (severe ROP) in different birth weight and gestational age groups were studied. Based on the results, the study aimed to review the existing screening criteria for ROP based on the highest birth weight and or gestational age of infants who developed ROP needing treatment (severe ROP).

\section{METHODS}

This is a retrospective descriptive study of infants with gestational age $\leq 34$ weeks and/ or birth weight $\leq 1750$ gm admitted in NICU of a tertiary care hospital from January, 2011 to December, 2015. The neonatal unit is a 60 bedded level III-B accredited unit with average annual admission of 1000 premature infants. Institutional ethics committee approval (IEC- NI/14/DEC/44/84) was obtained for conducting the study. Neonates with birth weight $\leq 34$ weeks and/or $\leq 1750$ gm admitted within 24 hours of birth and survived till the initial ROP screening were included. The infants who did not undergo the ophthalmological examination till complete vascularisation of retina or infants with major congenital ophthalmologic malformations, chorioretinitis or cataract were excluded from the study. Data were retrieved from medical records department using inpatient database classified according to international code of diseases-10 (ICD -10) using the ICD code "P-07.3" (code for preterm). It was cross checked with neonatal admission register maintained in the neonatal unit. Data pertaining to ROP including the LASER therapy was obtained from prospectively entered ROP registry maintained in the Ophthalmology department of our institute. The end point was either complete vascularization or need for laser therapy. The parameters including gestational age, birth weight and sex were collected. Gestational age was assigned by treating neonatologist according to the dating scan done between 6 and 8 weeks from last menstrual period (LMP) or if not available, from LMP. Infants were stratified into birth weight groups ( $<750 \mathrm{gm}$, 750-999 gm, 1000-1249 gm, 1250-1499 gm, $1500-1750 \mathrm{gm},>1750)$ and gestational age (GA) groups ( $\leq 28$ weeks, 29-30 weeks, 31-32 weeks, 33-34 weeks, $>34$ weeks ) which were based on the results of previous studies. This was done to enable ease of comparison with other national and international data.

In the neonatal unit, saturation limits of all preterm neonates requiring oxygen therapy or those on respiratory therapy were maintained strictly according to the protocol. During the study period of January 2011 to May 2013 and June 2013 to December 2015, oxygen targets were maintained between $88 \%$ and $92 \%$ and $91 \%$ and $95 \%$, respectively. The change in oxygen targets were based on the results of BOOST trial. ${ }^{17}$ All the staff nurses were trained to adjust $\mathrm{FiO}_{2}$ to maintain the target oxygen saturation. In addition, there were ongoing training sessions to reinforce the importance of maintaining target oxygen saturation.

In the present study, all the eligible infants were screened on day 21 of life, irrespective of the gestational age. Screening was performed by two senior ophthalmologists with more than 10 years of experience in ROP. Informed verbal consent was obtained from parents for ROP screening. The pupils were dilated using phenylephrine $(0.5 \%)$ and tropicamide (1\%) eye drops, 30 minutes prior to the procedure. During the procedure, procedural pain protocol was followed and vitals were monitored. The eye examination was undertaken with strict aseptic precautions using a binocular indirect ophthalmoscope with the use of eyelid specula and scleral depressors as necessary, under topical anesthesia (2\% proparacaine drops). The International Classification of ROP (ICROP) was used to document the ROP status of the baby, including stage, zone and extent of disease and presence or absence of plus disease. ${ }^{18}$ Subsequent follow-up eye examinations were done based on the zone and severity of ROP as per AAP recommendation. ${ }^{9}$ Infants who developed threshold disease as per ICROP classification, APROP in zone 1 and those who had pre-threshold disease as per ET-ROP guidelines were treated with LASER photocoagulation using $532 \mathrm{~nm}$ double frequency yag laser (green) with laser indirect ophthalmoscope. ${ }^{4,18}$ The avascular retina beyond the ridge was ablated using confluent medium intensity burns in one or more sessions. Laser therapy was done as early as possible within 3 days of identification of the eligible infants for therapy.

Data including stage and zone of ROP and details regarding therapy including laser photocoagulation were collected. The highest stage of ROP in either eye for an individual was recorded as the stage of ROP. In this study, severe ROP was defined as any stage of ROP needing treatment. 


\section{Outcome measures}

The outcome measures were the proportion of infants who developed ROP and severe ROP among the eligible infants. The incidence of ROP in various birth weight groups and gestational age were calculated. The largest birth weight and or the highest gestational age of infants who developed severe ROP were ascertained and taken as cut off points for revising the existing screening criteria for ROP. Screening criteria for ROP used in USA and UK were applied on the existing data, to compare the applicability of other national criteria with present study group.

\section{Sample size}

A sample size of 1250 was needed to calculate the incidence in our study population assuming a known incidence of $11.8 \%$ of any ROP and expected incidence of $15 \%$ with power of $80 \%$ and alpha error of $1 \%{ }^{24}$ The expected incidence of any ROP of $15 \%$ was from previous one year data (unpublished) of our institute.

\section{Data analysis}

The collected data was subjected to statistical analysis using Statistical Package for social sciences (SPSS 17.0; SPSS Inc, Chicago, IL). Scatter plot was used to represent the distribution of ROP and severe ROP and to decide the cut off for ROP screening for GA and birth weight. Chi-square test was performed to compare categorical data. The ' $p$ ' value of less than 0.05 was considered as statistically significant.

\section{RESULTS}

During the study period, there were a total of 1458 infants who were $\leq 34$ weeks and/or $\leq 1750$ gm. Figure 1 depicts the flow chart of the study population. Among 1366 infants included in this study, $53 \%$ were males. The mean gestational age (GA) was $31.2 \pm 2.2$ weeks. The mean birth weight (BW) was $1431 \pm 386.5 \mathrm{gm}$.

ROP was seen in 252 infants which accounts for an overall incidence of $18.4 \%$. Mean BW and GA of infants with ROP is depicted in Table 1 . The frequency distribution among infants with stage I, II and III ROP were $34 \%, 36 \%$ and $30 \%$, respectively. One infant progressed to stage IV in spite of treatment. The progression of ROP from stage I directly to stage III occurred in $7 \%$ of the infants.

The incidence of ROP and the distribution of stages of ROP according to birth weight groups are depicted in Table 2. Stratified analysis showed a significant increase in the incidence of ROP with decreasing birth weight (Chi-square test $\mathrm{p}<0.001)$. The incidence of ROP among 272 extremely low birth weight (ELBW) infants in the study cohort was $52.4 \%$ and among 893 very low birth weight (VLBW) infants was 28.5\%. Stage III occurred more frequently in lower birth weight group infants.

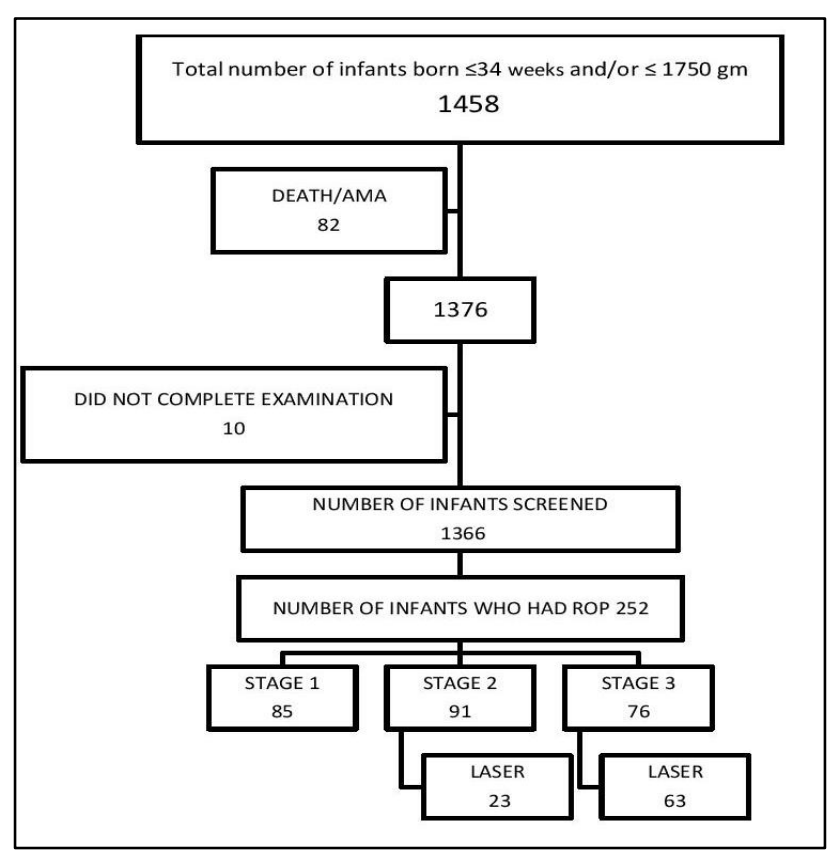

Figure 1: Flow chart of the study.

Table 1: Baseline characteristics of study group.

\begin{tabular}{|llll|}
\hline GA and BW & $\begin{array}{l}\text { Total } \\
\text { infants } \\
\text { screened }\end{array}$ & $\begin{array}{l}\text { Infants } \\
\text { with } \\
\text { ROP }\end{array}$ & $\begin{array}{l}\text { Infants } \\
\text { with } \\
\text { severe } \\
\text { ROP }\end{array}$ \\
\hline $\begin{array}{l}\text { Mean GA } \\
\text { (in weeks) }\end{array}$ & $31.2 \pm 2.2$ & $29.2 \pm 1.4$ & $28.7 \pm 2.3$ \\
\hline $\begin{array}{l}\text { GA in } \\
\text { weeks(range) }\end{array}$ & $24-36$ & $24-36$ & $24-35$ \\
\hline $\begin{array}{l}\text { Mean BW } \\
\text { (in gram) }\end{array}$ & $1431 \pm 386.5$ & $1120 \pm 233$ & $1022 \pm 287$ \\
\hline $\begin{array}{l}\text { BW in grams } \\
\text { (range) }\end{array}$ & $430-3315$ & $430-2250$ & $430-1800$ \\
\hline
\end{tabular}

GA-gestational age; BW-birth weight

The incidence of ROP and the distribution of the various stages of ROP according to GA groups are shown in Table 3. Stratified analysis showed a significant increase in the occurrence of ROP with lower GA (chi square test $\mathrm{p}<0.001)$. Incidence of ROP in infants with gestational age $\leq 28$ weeks was $60.8 \%$ and in infants with GA $\leq 32$ weeks was $23.8 \%$. None of the infants of GA greater than 36 weeks developed ROP.

The overall incidence of severe ROP (needing laser therapy) in present study was $6.2 \%$. In the entire cohort, laser treatment was done in 86 infants, of whom 63 infants had stage 3 ROP and 23 infants had stage 2 ROP. APROP was noted in two infants and 6 had plus disease. The frequency distribution of infants needing laser according to birth weight and GA are shown in Table 2 and Table 3 , respectively. 
One fourth of infants less than 28 weeks who developed ROP required treatment. More than $40 \%$ of infants of birth weight less than $750 \mathrm{gm}$ with ROP needed therapy.
There were no laser related adverse effects in the study group.

Table 2: Distribution of ROP in different birth weight groups.

\begin{tabular}{|lllllll|}
\hline $\begin{array}{l}\text { Birth weight in } \\
\text { gram }\end{array}$ & $\begin{array}{l}\text { Total number } \\
\text { screened }\end{array}$ & $\mathbf{R O P}(\mathbf{n} \%)$ & Stage 1 (n \%) & Stage 2 (n \%) & Stage 3 (n \%) & Laser (n \%) \\
\hline$<750$ & 27 & $18(66.7)$ & $1(3.7)$ & $8(29.6)$ & $9(33.3)$ & $12(44.4)$ \\
\hline $750-999$ & 145 & $79(54.4)$ & $25(17.2)$ & $25(17.2)$ & $29(20)$ & $30(20.7)$ \\
\hline $1000-1249$ & 285 & $79(27.7)$ & $27(9.4)$ & $30(10.5)$ & $22(7.7)$ & $23(8.1)$ \\
\hline $1250-1499$ & 303 & $41(13.5)$ & $17(5.6)$ & $16(5.2)$ & $8(2.6)$ & $13(4.3)$ \\
\hline $1500-1750$ & 349 & $23(6.6)$ & $9(2.3)$ & $8(2.4)$ & $6(1.7)$ & $6(1.7)$ \\
\hline$>1750$ & 257 & $12(4.7)$ & $6(2.3)$ & $4(1.5)$ & $2(0.7)$ & $2(0.7)$ \\
\hline
\end{tabular}

The distribution of infants with ROP and severe ROP with GA plotted on $\mathrm{x}$-axis and birth weight plotted on $y$-axis is depicted in the Figure 2. If screening criteria practiced in USA (GA < 30 weeks and $\mathrm{BW}<1500$ grams) is followed, $10.7 \%$ of infants with ROP and $11.6 \%$ with severe ROP would have been missed. If screening criteria of $\mathrm{UK}$ (GA $<32$ weeks and $\mathrm{BW}<1500$ grams) is followed, $8.3 \%$ of infants with ROP and $9.3 \%$ with severe ROP would have been missed. A threshold of BW $\leq 1750 \mathrm{gm}$ and or $\leq 32$ weeks would not miss any infant with severe ROP and would miss only $3(1.1 \%)$ infants with ROP (not needing treatment). All the 3 infants were of GA 33 weeks, 2 with birth weight 1900 grams and one with 2250 grams. There are 133 infants above this cut off who were screened and did not develop severe ROP.

Table 3: Distribution of ROP in different gestational age groups.

\begin{tabular}{|lllllll|}
\hline GA weeks & $\begin{array}{l}\text { Total number } \\
\text { screened }\end{array}$ & $\mathbf{R O P +}(\mathbf{n} \%)$ & Stage 1 (n \%) & Stage 2 (n \%) & Stage $\geq \mathbf{3}(\mathbf{n} \%)$ & Laser (n \%) \\
\hline$\leq 28$ weeks & 171 & $104(60.8)$ & $20(11.6)$ & $45(26.3)$ & $39(22.8)$ & $44(25.7)$ \\
\hline 29-30 weeks & 283 & $75(26.5)$ & $33(11.6)$ & $24(8.4)$ & $18(6.4)$ & $21(7.4)$ \\
\hline $31-32$ weeks & 506 & $50(9.8)$ & $22(4.3)$ & $15(2.9)$ & $13(2.6)$ & $16(3.1)$ \\
\hline $33-34$ weeks & 342 & $18(5.2)$ & $8(2.3)$ & $6(1.7)$ & $4(1.2)$ & $3(0.9)$ \\
\hline$>34$ weeks & 64 & $5(7.6)$ & $2(3.0)$ & $1(1.5)$ & $2(3.1)$ & $2(3.1)$ \\
\hline
\end{tabular}

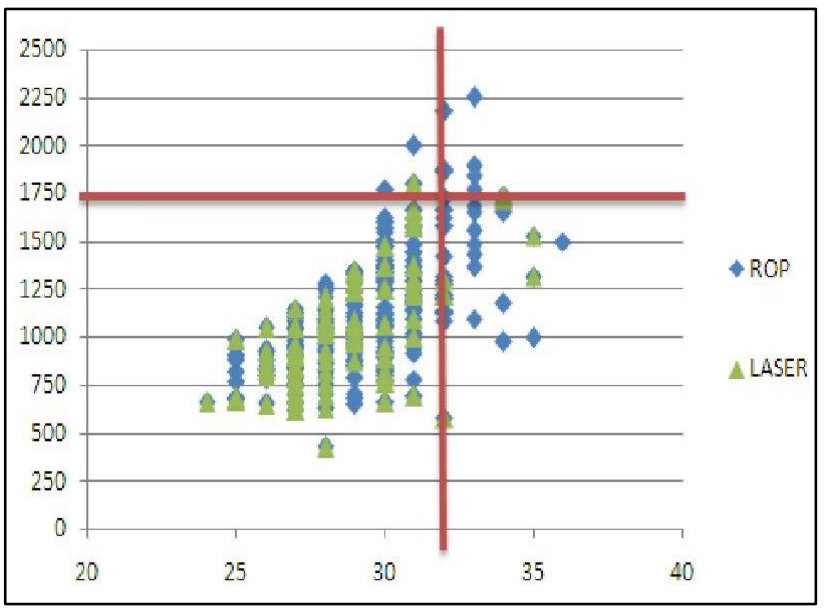

Figure 2: Scatter plot of distribution of infants with ROP and severe ROP with GA plotted on $\mathrm{X}$-axis and birth weight plotted on $\mathrm{Y}$ - axis.

\section{DISCUSSION}

With increasing survival of extreme preterm infants, ROP has become an emerging global problem and hence screening is important to prevent blindness. Aggressive posterior ROP (APROP) can occur early in very low birth weight (VLBW) infants and has poor visual outcome which is relatively common in Indian infants. ${ }^{19,20}$ Therefore in the present study, initial screening of eligible infants admitted in neonatal unit was undertaken at 3 weeks of life irrespective of gestational age. Incidence in ROP in our study was $18.3 \%$. Incidences of ROP in various studies in India have been reported to be $51 \%$ from Delhi (79 patients), $47 \%$ in a study from Chandigarh (165 patients), 44\% from north east (50 patients), $38 \%$ from Chennai (50 patients), $22 \%$ from Bangalore (7106 images), 22.3\% from Pune (552 infants) and $11.8 \%$ from AIIMS, Delhi (704 patients). ${ }^{6-8,21-24}$ Among the various studies done in India, the present 
study involved the highest number of infants screened for ROP. The incidences of ROP in other parts of the world are $12.7 \%$ in China (472 patients), 34\% in Egypt (152 patients) and 56\% in Saudi Arabia (174 patients). ${ }^{25-27}$ Our study has concurred with other national and international studies in showing an independent association of GA and BW with ROP. ${ }^{5,13,21,23-27}$

The incidence of ROP among VLBW infants was $28.5 \%$, and among ELBW infants was $52.4 \%$ which are comparable to the incidence reported from various studies done internationally (Figure 3). ${ }^{4,5,28-32}$ Maintaining a strict protocol for oxygenation in our unit could be the reason for the comparable incidence of ROP in VLBW and ELBW infants to that of international data. The incidence of severe ROP (treated with laser) in the present study was $6.2 \%$ among the infants screened. Among those who developed ROP, severe ROP occurred in $34.1 \%$ and in literature the incidence varied from 16.9 to $63.6 \%$ (Figure 3)..$^{4,5,28-32}$ However, compared to developed nations, we had bigger infants with the highest BW being 2250 in ROP group and 1800 in severe ROP group and more mature infants with highest GA of 36 weeks in ROP group and 35 weeks in severe ROP group.

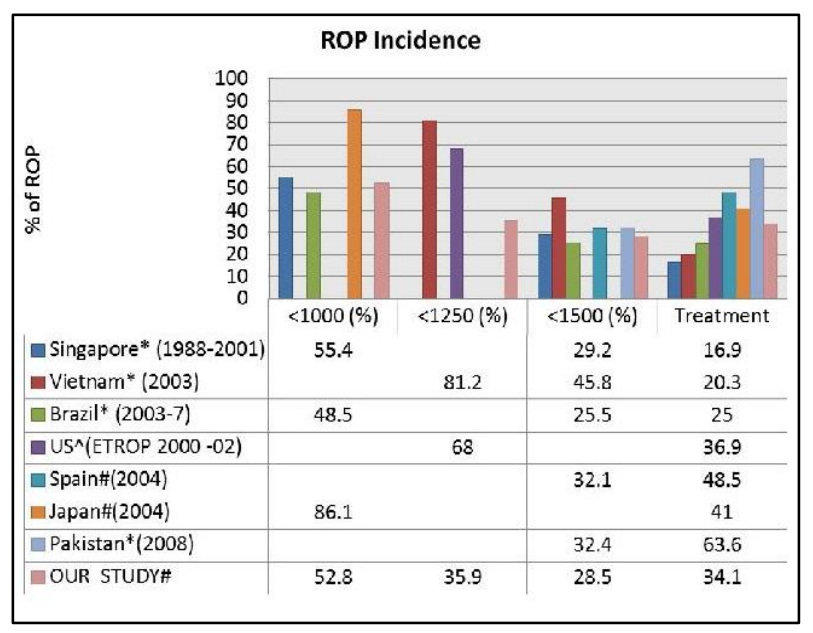

*Threshold ROP among infants with ROP

${ }^{\wedge}$ Prethreshold needing treatment among infants with ROP \#Threshold/prethreshold needing treatment among infants with ROP.

Figure 3: Comparison of the incidence of ROP and severe ROP in present study with international data in various birth weight groups.

There are wide variation in proportion and incidence of severe ROP. More mature infants develop ROP in low and middle income countries. ${ }^{1,14}$ This could be due to difference in the management protocol in neonatal units or ethnicity or genetic polymorphism. ${ }^{33}$ With ultimate aim to prevent childhood blindness due to ROP, the focus should be not to miss any infant with severe ROP. With the existing resources and the expertise, it is also equally important not to over burden the system. As the study group varies with time, revising the screening protocol from time to time is a good practice. The other methods like the WINROP algorithm has not yet been proven to sensitively pick up infants needing treatment. ${ }^{34} \mathrm{BW}$ and GA remain the standard risk factors. $5,13,21,23-27$

Screening criteria with $\mathrm{BW} \leq 1750$ gms and or GA $<32$ weeks would detect all the infants needing treatment (severe ROP) and would miss only $3(1.1 \%)$ infants with ROP who did not need treatment. This cut off would avoid unnecessary examination of 133 infants. In the presence of additional risk factors and at the discretion of treating neonatologists, infants with GA >32 weeks and BW >1750 grams should continue to be screened. Screening criteria of USA and UK cannot be applied to Indian population as it would have missed a significant proportion of infants with severe ROP.

The study involved large sample, with distribution across varied GA and BW which is more representative of the general population. This is a single institutional study with standardized protocol being practiced in the neonatal unit. Birth weight and GA was ascertained accurately. The limitations are the retrospective study design and that risk factors were not analysed. Further research is needed to find out if combining the risk factors with GA and BW for more mature infants would bring down the cut off for screening. Those infants with BW above 1750 and GA $>34$ weeks with a turbulent course though screened were not included in the study. There is a need for further larger population based study or multi-institutional study to refine the screening criteria for Indian infants.

\section{CONCLUSION}

In the present study, incidence of ROP and severe ROP were $18.4 \%$ and $6.2 \%$, respectively among premature infants with GA $<34$ weeks and or BW $\leq 1750 \mathrm{gm}$. Applying screening criteria of developed nations for the present study cohort, would miss 9.3 to $11.6 \%$ of infants with severe ROP. We suggest infants born premature with GA of $\leq 32$ and or BW of $\leq 1750$ gm weeks should undergo screening for ROP, as this screening criteria has detected all infants with severe ROP.

Funding: No funding sources Conflict of interest: None declared

Ethical approval: The study was approved by the Institutional Ethics Committee

\section{REFERENCES}

1. Blencowe H, Lawn JE, Vazquez T. Pretermassociated visual impairment and estimates of retinopathy of prematurity at regional and global levels for 2010. Pediatr Res. 2013;74(1):35-49.

2. Zin A, Gole GA. Retinopathy of prematurityincidence today. Clin Perinatol. 2013;40:185-200.

3. Clare G, Allen F. Childhood blindness in the context of VISION 2020-the right to sight. Bulletin World Health Organization. 2001;79:227-32. 
4. Early Treatment for retinopathy of prematurity cooperative group revised indications for the treatment of retinopathy of prematurity: results of the early treatment for retinopathy of prematurity randomized trial. Arch Ophthalmol. 2003;121:168494.

5. Shah VA, Yeo CL, Ling YL, Ho LY. Incidence, risk factors of retinopathy of prematurity among very low birth weight infants in Singapore. Ann Acad Med Singapore. 2005;34(2):169-78.

6. Charan R, Dogra MR, Gupta A, Narang A. The incidence of retinopathy of prematurity in a neonatal care unit. Indian J Ophthalmol. 1995;43:123-6.

7. Gopal L, Sharma T, Ramchandran S, Shanmugasundaram R, Asha V. Retinopathy of prematurity. Indian J Ophthalmol. 1995;43:50-61.

8. Varughese S, Jain S, Gupta N, Singh S, Tyagi V, Puliyel JM. Magnitude of the problem of retinopathy of prematurity experience in a large maternity unit with a medium size level-3 nursery. Indian J Ophthalmol. 2001:49:187-8.

9. Fierson WM. Screening examination of premature infants for retinopathy of prematurity. Pediatrics. 2013;131:189-95.

10. Retinopathy of prematurity: guidelines for screening and treatment report of a joint working party of the royal college of ophthalmologists and British association of perinatal medicine. Early Hum Dev. 1996;46:239-58.

11. Lee SK, Normand C, Mcmillan D. Evidence for changing guidelines for routine screening for retinopathy of prematurity. Arch Pediatr Adolesc Med. 2001;155:387-95.

12. Jefferies A. Retinopathy of prematurity: recommendations for screening. Retin Paediatr Child Health. 2010;15:667-74.

13. Akcakaya AA, Yaylali SA, Erbil HH. Screening for retinopathy of prematurity in a tertiary hospital in Istanbul: incidence and risk factors. $\mathrm{J}$ Pediatr Ophthalmol Strabismus. 2012;49:21-5.

14. Jalali S, Matalia J, Hussain A. Modification of screening criteria for retinopathy of prematurity in India and other middle-income countries. Am J Ophthalmol. 2006;141:966-8.

15. Chen Y, Li X. Characteristics of severe retinopathy of prematurity patients in China: a repeat of the first epidemic? Br J Ophthalmol. 2006;90:268-71.

16. Pejawar R, Vinekar A, Bilagi A. National neonatology forum evidence-based clinical practice guidelines (2010), retinopathy of prematurity. NNF India, New Delhi. 2010:253-62.

17. Stenson BJ, Tarnow WO, Darlow BA, Simes J, Juszczak E, Askie L. Oxygen saturation and outcomes in preterm infants. $N$ Engl $J$ Med. 2013;368(22):2094-104.

18. International committee for the classification of retinopathy of prematurity. The International classification of retinopathy of prematurity revisited. Arch Ophthalmol. 2005;123:991-9.
19. Sanghi G, Dogra MR, Das P, Vinekar A, Gupta A, Dutta S. Aggressive posterior retinopathy of prematurity in Asian Indian babies: spectrum of disease and outcome after laser treatment. Retina. 2009;29(9):1335-9.

20. Shah P, Narendran V, Saravanan VR, Raghuram A. Fulminate retinopathy of prematurity-clinical characteristics and laser outcome. Indian J Ophthalmol. 2005;53:261-5.

21. Chattopadhyay MP, Pradhan A, Singh R, Datta S. Incidence and risk factors for retinopathy of prematurity in neonates. Indian Pediatr. 2015;52(2):157-8.

22. Vinekar A, Gilbert C, Dogra M, Kurian M, Shainesh G, Shetty B. The kidrop model of combining strategies for providing retinopathy of prematurity screening in underserved areas in India using widefield imaging, tele-medicine, non-physician graders and smart phone reporting. Indian $\mathrm{J}$ Ophthalmol. 2014;62(1):41-9.

23. Chaudhari S, Patwardhan V, Vaidya U, Kadam S, Kamat A. Retinopathy of prematurity in a tertiary care center, risk factors and outcome. Indian Pediatr. 2009;46:219-24.

24. Kumar P, Sankar MJ, Deorari A, Azad R, Chandra P, Agarwal R, et al. Risk factors for severe retinopathy of prematurity in preterm low birth weight neonates. Indian J Pediatr. 2011;78:812-6.

25. Chen Y, Xun D, Wang YC, Wang B, Geng SH, Chen $\mathrm{H}$, et al. Incidence and risk factors of retinopathy of prematurity in two neonatal intensive care units in North and South China. Chin Med J. 2015;128(7):914-8.

26. Hadi AM, Hamdy IS. Correlation between risk factors during the neonatal period and appearance of retinopathy of prematurity in preterm infants in neonatal intensive care units in Alexandria, Egypt. Clin Ophthalmol. 2013;7:831-7.

27. Binkhathlan AA, Almahmoud LA, Saleh MJ. Retinopathy of prematurity in Saudi Arabia: incidence, risk factors, and the applicability of current screening criteria. $\mathrm{Br} \mathrm{J}$ Ophthalmol. 2008;92:167-9.

28. Phan MH, Nguyen PN, Reynolds JD. Incidence and severity of retinopathy of prematurity in Vietnam, a developing middle-income country. J Pediatr Ophthalmol Strabismus. 2003;40(4):208-12.

29. Fortes JB, Eckert GU, Valiatti FB, Costa MC, Bonomo PP, Procianoy RS, et al. Prevalence of retinopathy of prematurity: an institutional crosssectional study of preterm infants in Brazil. Rev Panam Salud Publica. 2009;26(3):216-20.

30. Taqui AM, Syed R, Chaudhry TA, Ahmad K, Salat MS. Retinopathy of prematurity: frequency and risk factors in a tertiary care hospital in Karachi, Pakistan. J Pak Med Assoc. 2008;58(4):186-90.

31. Hernandez M, Orduna C, Bosch V, Salinas R, Alcaraz JL, Marin JM. Retinopathy of prematurity in the Murcia region of Spain incidence and severity. Arch Soc Esp Oftalmol. 2008;83(7):423-8. 
32. Hiraoka M, Watanabe $\mathrm{T}$, Kawakami $\mathrm{T}$, Ito $\mathrm{R}$, Takigawa I, Suzumura H, et al. Retinopathy of prematurity in extremely low birth weight infants: a Tokyo multicenter study. Nippon Ganka Gakkai Zasshi. 2004;108(10):600-5.

33. Ortega JM, Anaya AR, Uberos FJ. Genetic and environmental influences on retinopathy of prematurity. Mediators Inflamm.2015;2015:764159.
34. Lundgren P, Stoltz SE, Domell M. Winrop identifies severe retinopathy of prematurity at an early stage in a nation-based cohort of extremely preterm infants. PLoS ONE. 2013;8:73256.

Cite this article as: Umamaheswari B, Shafi JSM, Manian N, Muthaya M, Thomas M, Amboiram P, et al. Screening based on incidence of severe retinopathy of prematurity in a tertiary care center in India: are Indian infants different? Int J Contemp Pediatr 2016;3:847-53. 